Website: http://revistas.lamolina.edu.pe/index.php/acu/index

(C) Universidad Nacional Agraria La Molina, Lima - Perú

\title{
Disponibilidad de forraje en el pasto castilla (Panicum maximum Jacq.) según intervalos de corte y crecimiento estacional en una zona costera
}

\author{
Forage availability in pasture castilla (Panicum maximum Jacq.) According to intervals of cut and \\ seasonal growth in a coastal area
}

Fernando Jesús Passoni Telles ${ }^{1 *}$, Javier Arias Carbajal ${ }^{2}$ y Edgardo Arturo Vilcara Cárdenas ${ }^{3}$

* Autor de correspondencia

\section{Resumen}

El presente trabajo tuvo como objetivo evaluar el efecto de intervalos de corte en la producción de forraje del pasto Castilla (Panicum maximun Jacq.) en verano e invierno de una zona de la costa, así como estimar la carga animal. Fue realizado, entre diciembre de 2015 y septiembre de 2016, en el Campo Experimental El Tomatillo" del Programa de Investigación y Proyección Social en Pastos y Forrajes de la Universidad Nacional Agraria La Molina. Para el análisis estadístico, se utilizó un diseño completamente al azar y tres repeticiones para evaluar cuatro edades de corte $(3,6,9$ y 12 semanas de rebrote) en la estación de verano e invierno, respectivamente. No se aplicó fertilización. Las 12 parcelas de $4 \mathrm{~m}^{2}$ cada una fueron uniformizadas mediante un corte general al inicio de cada estación. Las muestras de forraje fueron obtenidas cortando $1 \mathrm{~m}^{2}$ en cada parcela, según la edad de rebrote, se pesaron y se registraron los datos en formatos para su posterior procesamiento. Los resultados indican que, tanto en verano como invierno, la producción de forraje disponible fue ascendente conforme avanzaba la edad de corte. El análisis de varianza mostró diferencias significativas $(\mathrm{P}>0.05)$ entre edades de rebrote en el verano más no así en el invierno. En ambos períodos estacionales se demostró la estrecha relación que existe entre la variable intervalo de corte, disponibilidad de forraje y estimación de carga animal. La disponibilidad de forraje verde en verano fue superior a la de invierno y es explicado por ser un pasto adaptado a condiciones tropicales y subtropicales. Asimismo, la estimación de la carga animal es mayor en verano y es explicado por la mayor disponibilidad del pasto en esta estación.

Palabras clave: Pasto Castilla (Panicum máximum Jacq.); intervalo de corte; disponibilidad de forraje; estación de crecimiento; estimación carga animal; zona costera.

\begin{abstract}
The objective of this study was to evaluate the effect of cutting intervals on forage production of the pasture Castilla (Panicum maximum Jacq.) in the summer and winter of a coastal area as well as estimating the animal load. It was carried out, between December 2015 and September 2016, in the Experimental Field "El Tomatillo" belonging to the Program of Research and Social Projection in Pastures and Forages of the National Agrarian University La Molina. We used 12 plots of $4 \mathrm{~m}^{2}$ each. For the statistical analysis, a completely randomized design and three repetitions were used to evaluate four cutting ages $(3,6,9$ and 12 weeks of regrowth) in the summer and winter season, respectively. No fertilization was applied. The 12 plots of $2 \mathrm{~m}^{2}$ each were uniformed by a general court at the beginning of each season. Forage samples were obtained by cutting $1 \mathrm{~m}^{2}$ in each plot, depending on the age of regrowth, were weighed and the weights were recorded in formats for later processing. The results indicated that in both summer and winter, forage production was ascending as the cutting age progressed. The variance analysis showing significant differences $(\mathrm{P}>0.05)$ between ages of regrowth in the summer but not in the winter. In both seasonal periods, the close relationship between the variable cutting interval, forage availability and animal estimation was demonstrated. The availability of green forage in summer was higher than that of winter and is explained by being a pasture adapted to tropical and subtropical conditions. Also, the estimation of the animal load is higher in summer and is explained by the greater availability of the pasture in this season.
\end{abstract}

Keywords: Pasture Castilla (Panicum maximum Jacq.); cutting interval; forage availability; growth station; animal load estimation; coastal area.

\section{Introducción}

Las explotaciones ganaderas lecheras en la costa peruana se sustentan mayoritariamente en la alimentación a base de maíz chala (Zea mays) y pasto elefante (Pennisetum purpureum). Una alternativa para incrementar el piso forrajero y contar con más diversidad de especies forrajeras sería la introducción del Pasto Castilla (Panicum maximum) el cual es una gramínea tropical-subtropical perenne de tallos erectos que crece muy bien hasta los $2000 \mathrm{msnm}$. Es muy resistente a la sequía, presenta gran

\footnotetext{
1,2,3 Departamento Académico de Fitotecnia, Facultad de Agronomía, Universidad Nacional Agraria La Molina, Apartado postal 12-056, La Molina, Lima, Perú. Emails: fpassoni@lamolina.edu.pe; ariascj@lamolina.edu.pe; eavilcara@lamolina.edu.pe
} 
valor nutritivo cuando es joven y es apetitoso por el ganado (Fernández, et al., 2004; Ramírez, et al., 2009; Zanini, et al., 2012).

El pasto Castilla se puede sembrar en la costa central de octubre a marzo y en la costa norte, todo el año. En la selva, ha sido sometido a evaluaciones mostrando un buen potencial como pasto de corte (Passoni et al., 1992). Desde hace buen tiempo, trabajos de investigación han demostrado que al aumentar el intervalo entre cortes se incrementa la producción de forraje verde del pasto Castilla (Oyenuga, 1960; Passoni et al., 1992; Reynoso et al., 2009; García et al., 2008; Zanini, et al., 2012). Sin embargo, se sostiene que el mejor momento de aprovechamiento del pasto por el ganado es cuando tiene una edad de siete a nueve semanas de rebrote, intervalo en el cual hay una cantidad adecuada de hojas, buen contenido proteico y baja fibra (Skerman y Riveros, 1992; Fernández, et al., 2004; Reynoso, et al., 2009). Finalmente, dadas las bondades demostradas del pasto Castilla se espera que, mediante un manejo adecuado, sirva como un forraje alternativo para la alimentación del ganado lechero que se explota en la costa de nuestro país.

Las razones expuestas condujeron a ejecutar el presente trabajo que tuvo como objetivos evaluar el efecto de la edad de corte en la disponibilidad de forraje verde del pasto Castilla (Panicum maximum Jacq.) en las estaciones de verano e invierno en condiciones de la costa central, así como estimar, en base al forraje producido, la carga animal a sostener por hectárea.

\section{Materiales y métodos}

El trabajo fue realizado en el Campo Experimental "El Tomatillo" perteneciente al Programa de Investigación y Proyección Social en Pastos y Forrajes de la Facultad de Agronomía de la Universidad Nacional Agraria La Molina. Las doce parcelas de $4.0 \mathrm{~m}^{2}$ cada una $(2 \times 2 \mathrm{~m})$ fueron cortadas por completo en dos momentos: el 22 de diciembre de 2015 (verano) y el 22 de junio de 2016 (invierno) para dar inicio a las evaluaciones de 3, 6, 9 y 12 semanas de rebrote en cada estación. Las muestras fueron tomadas de cuadrantes de $1,0 \mathrm{~m}^{2}$, el forraje cortado fue pesado en el campo, los datos anotados en formatos de evaluación, y colocado en bolsas de plástico. No se aplicó ningún fertilizante. Se empleó el diseño completamente al azar con cuatro tratamientos (edad de rebrote) y tres repeticiones. Se realizó un análisis de regresión para determinar la producción de forraje verde en función de la edad de corte del pasto. Para evaluar la producción de forraje verde en verano o invierno se usó el modelo matemático siguiente:

donde:

$$
Y_{i}=\mu+\alpha_{i}+\varepsilon_{i j}
$$

$\mathrm{Y}_{\mathrm{ij}}=$ es el valor de la producción de forraje verde a las 3, 6, 9 ó 12 semanas de rebrote en la i-ésima repetición

$\mu=$ media global

$\alpha_{i}=$ es el efecto del tratamiento $i, i=1 \ldots 4$

$\varepsilon_{\mathrm{ij}}=$ error experimental

\section{Resultados y discusión}

\section{Producción de forraje verde disponible}

La producción de forraje verde (FV) en kilos por metro cuadrado $\left(\mathrm{kg} . \mathrm{m}^{-2}\right)$ del pasto Castilla, en verano, se aprecia en la Tabla 1. Los rendimientos promedios fueron de 0,351; 0,$589 ; 1,058$ y $1,128 \mathrm{~kg} \mathrm{FV} \mathrm{m}^{-2}$ a las 3, 6, 9 y 12 semanas de rebrote, respectivamente siendo 66,$95 ; 77,25 ; 84,45$ y $82 \%$ más altos que el período de invierno $(0,116 ; 0,134$; 0,164 y $0,203 \mathrm{~kg} \mathrm{FV} \mathrm{m}^{-2}$, respectivamente). De acuerdo con el análisis de varianza (ANOVA), hubo diferencias significativas $(\mathrm{P}>0.05)$ entre edades de rebrote en el verano tal como se muestra en la Tabla 2.

Tabla 1. Producción media de forraje verde $\left(\mathrm{kg} \mathrm{m}^{-2}\right)$ a diferentes edades de corte del Pasto Castilla en la estación de verano

\begin{tabular}{ccccc}
\hline \multirow{2}{*}{ Repeticiones } & \multicolumn{5}{c}{ Edad de rebrote (semanas) } \\
& 3 & 6 & 9 & 12 \\
\hline Media & 0,351 & 0,589 & 1,058 & 1,128 \\
D. Estándar & 0,346 & 0,1635 & 0,3434 & 0,3471 \\
\hline
\end{tabular}

Tabla 2. Anova para la producción de forraje verde $\left(\mathrm{kg} \mathrm{m}^{-2}\right)$ del pasto castilla en el período de verano

\begin{tabular}{lccccc}
\hline \multicolumn{1}{c}{ Fuente } & $\begin{array}{c}\text { Grados } \\
\text { de } \\
\text { libertad }\end{array}$ & $\begin{array}{c}\text { Suma de } \\
\text { cuadrados }\end{array}$ & $\begin{array}{c}\text { Cuadrado } \\
\text { Medio }\end{array}$ & Razón-F & Valor-P \\
\hline Tratamiento & 3 & 1,2576416 & 0,419214 & 6,296 & 0,01682 \\
Error & 8 & 0,5326693 & 0,066583 & & \\
Total & 11 & 1,7903109 & & & \\
\hline
\end{tabular}

En la estación de invierno, el pasto Castilla mostró menores rendimientos de forraje verde a pesar de mantener una producción promedio ascendente de 0,$117 ; 0,134$; 0,164 y $0,203 \mathrm{~kg} \mathrm{FV} \mathrm{m}^{-2}$ a las 3, 6, 9 y 12 semanas de rebrote (Tabla 3 ).

Tabla 3. Producción de forraje verde $\left(\mathrm{kg} \mathrm{m}^{-2}\right)$ a diferentes edades de corte del Pasto Castilla en la estación de invierno

\begin{tabular}{ccccc}
\hline \multirow{2}{*}{ Repeticiones } & \multicolumn{4}{c}{ Edad de rebrote (semanas) } \\
& 3 & 6 & 9 & 12 \\
\hline Media & 0,117 & 0,134 & 0,164 & 0,203 \\
D. Estándar & 0,0358 & 0,0294 & 0,0348 & 0,0557 \\
\hline
\end{tabular}

El análisis de varianza (Tabla 4) indicó que no existe una diferencia significativa entre las medias de las cuatro edades de rebrote con un nivel del 5\% de significación (el valor-P de la razón-F es mayor o igual que 0,05 ).

Tabla 4. Anova para la producción de forraje verde $\left(\mathrm{kg} \mathrm{m}^{-2}\right)$ del pasto castilla en el período de invierno

\begin{tabular}{lccccc}
\hline \multicolumn{1}{c}{ Fuente } & $\begin{array}{c}\text { Grados de } \\
\text { libertad }\end{array}$ & $\begin{array}{c}\text { Suma de } \\
\text { cuadrados }\end{array}$ & $\begin{array}{c}\text { Cuadrado } \\
\text { Medio }\end{array}$ & Razón-F Valor-P \\
\hline Tratamiento & 3 & 0,0128609 & 0,0042869 & 2,652 & 0,1200 \\
Error & 8 & 0,0129300 & 0,0016162 & & \\
Total & 11 & 0,0257909 & & & \\
\hline
\end{tabular}


Los promedios de producción de forraje verde en cada intervalo de tres semanas fueron diferentes entre estaciones. Se ajustaron ecuaciones de regresión cuadrática para las dos estaciones (verano e invierno) entre la producción de forraje verde y la edad de corte o rebrote.

Los valores más altos se registraron a las nueve y 12 semanas de rebrote siendo mayor la producción durante el verano (Tabla 5; Figura 1). Lo anterior indica que el pasto Castilla responde en forma diferente a las condiciones ambientales al ser un pasto tropical que se desarrolla muy bien a alta temperatura mas no así en el invierno cuando esta baja (Sánchez y Salinas, 1993; Gerdes, et al., 2000; Ruiz, et al., 2015).

Tabla 5. Comparación entre verano e invierno de la producción de forraje $\left(\mathrm{kg} \mathrm{m}^{-2}\right)$ a diferentes edades de corte

\begin{tabular}{ccccc}
\hline $\begin{array}{c}\text { Edad de corte } \\
\text { (semanas) }\end{array}$ & Verano & Invierno & Promedio & DE \\
\hline 3 & 0,351 & 0,117 & 0,234 & 0,165 \\
6 & 0,589 & 0,134 & 0,361 & 0,322 \\
9 & 1,058 & 0,164 & 0,611 & 0,632 \\
12 & 1,128 & 0,203 & 0,665 & 0,654 \\
\hline
\end{tabular}

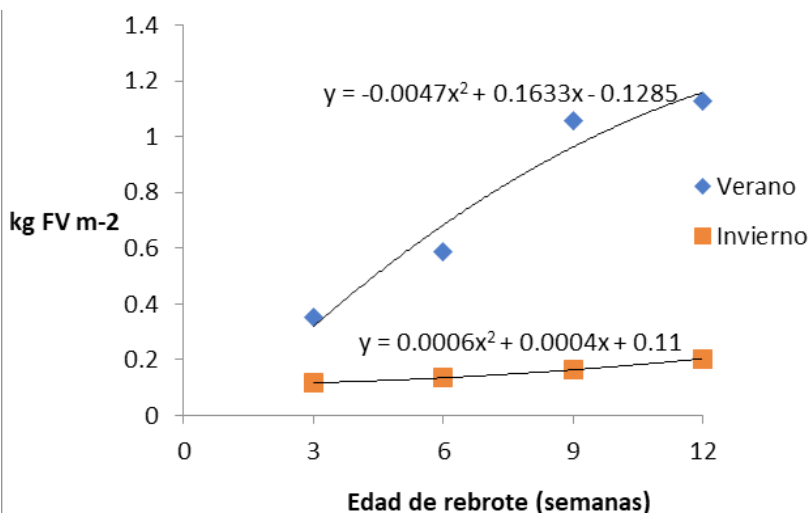

Figura 1. Rendimiento de forraje verde del Pasto Castilla según edad de rebrote y estación del año

Al avanzar la edad del pasto Castila se incrementó la producción de forraje, lo cual se puede deber al aumento del proceso fotosintético y con ello la síntesis de carbohidratos estructurales, influyendo de forma directa los factores climáticos predominantes en el verano donde las altas temperaturas, favorecen los procesos fisiológicos de la planta. Por otro lado, la disminución en la producción durante la estación de invierno se debe a que las bajas temperaturas limitan la eficiencia en cuanto a los procesos fisiológicos y, por lo tanto, el desarrollo de la planta lo cual es explicado por Herrera, 2006 y Zanini et al., 2012.

\section{Forraje disponible y carga animal}

El pasto Castilla o cualquier otro de corte, debe suministrarse con una edad de rebrote entre 60 y 70 días donde presenta una adecuada producción de forraje y calidad (Skerman y Riveros, 1992; Passoni et al., 1992; Fernández et al., 2004). En el presente ensayo, el Pasto Castilla, con una edad de rebrote de 63 días produjo en verano e invierno 1,058 y $0,164 \mathrm{~kg}$ de $\mathrm{FV} \mathrm{m}^{-2}$, respectivamente. Para efectuar algunas estimaciones, un vacuno adulto tiene una capacidad de consumo del $10 \%$ de su peso vivo más un $5 \%$ de pérdidas del pasto por su manipulación; es decir, un animal de 400 $\mathrm{kg}$ de peso vivo requiere de $60 \mathrm{~kg}$ de forraje verde por día. Con estos datos se puede estimar la carga animal expresada en vacunos por hectárea por día (Tabla 6).

\section{Conclusiones}

Los resultados establecieron la estrecha relación que existe entre la variable edad de corte y el rendimiento, impactando de manera significativa en el verano más no así en el invierno. El decrecimiento en la producción de forraje disponible durante la estación de invierno fue mayor debido a las bajas temperaturas en las cuales no responde el pasto Castilla. Ha quedado demostrado que, si la edad de corte aumenta, los rendimientos de forraje verde por hectárea para el pasto Castilla también aumentan, aunque en menor cantidad en el invierno. La carga animal estimada aumentará en la medida que aumente la producción de forraje disponible del pasto Castilla para lo cual se recomienda un buen plan de manejo que incluya fertilización y corte cada 60 días en promedio.

\section{Literatura citada}

Fernández, J.; Benítez, D.; Gómez, I.; de Souza, A. y Espinoza, R. 2004. Rendimiento de materia seca y contenido de proteína bruta del pasto Panicum maximum vc. Likoni en un suelo vertisol de la provincia de Granma, Cuba. Revista Cubana de Ciencias Agrícolas, Tomo 38 (4).

Garcia, C.; Martínez, R.; Tuero, R.; Cruz, A. 2008. Evaluación agronómica de Guinea Mombaza (Panicum maximum Jacq) en un suelo ferralítico rojo típico de la provincia La Habana, Cuba. Revista Cubana de Ciencias Agrícolas, Tomo 42 (2).

Gerdes, I.; Werner, c.; Colozzo, T.; Carvalho, D. y Schammass, A. 2000. Avaliacao de características agronómicas e morfológicas das gramineas forrageiras Marandu, Setaria e Tanzania aos 35 dias de crescimento mas estacoes do ano. Revista Brasileira de Zootecnia 29(4):947-954.

Tabla 6. Estimación de la carga animal en base al forraje disponible ofrecido por el pasto Castilla a las 9 semanas de rebrote

\begin{tabular}{|c|c|c|c|c|c|c|c|}
\hline Estación & Área $\left(\mathrm{m}^{2}\right)$ & Días & $\mathrm{m}^{2} \mathrm{~d}^{-1}$ & $\begin{array}{c}\text { Forraje } \\
\text { disponible }(\mathrm{kg} \\
\left.\mathrm{m}^{-2}\right)\end{array}$ & $\mathrm{kg} \mathrm{FV} \mathrm{d}^{-1}$ & $\begin{array}{l}\text { Demanda por } \\
\text { animal }\left(\mathrm{kg} \mathrm{d}^{-1}\right)\end{array}$ & $\begin{array}{l}\text { Estimación carga animal } \\
\left(\text { vacunos } \mathrm{ha}^{-1} \mathrm{~d}^{-1}\right)\end{array}$ \\
\hline Verano & 10000 & 63 & 158,73 & 1,058 & 167,94 & 60 & 2,80 \\
\hline Invierno & 10000 & 63 & 158,73 & 0,164 & 26,03 & 60 & 0,43 \\
\hline
\end{tabular}


Guerra, C.; Cabrera, A. y Fernández, L. 2003. Criterios para la selección de modelos estadísticos en la investigación. Revista Cubana de Ciencias Agrícolas 37(1):3-10.

Gutiérrez, H. y De la Vara, R. 2004. Análisis y diseños de experimentos. Editorial EDICUSA, México, D.F.

Herrera, R. 2006. Fotosíntesis. En: Pastos tropicales, contribución a la fisiología, establecimiento, rendimiento de biomasa, producción de biomasa, producción de semillas y reciclaje de nutrientes. Ed. EDICA, ICA. La Habana, Cuba.

Oyenuga, V. A. 1960. Effect of stage of growth of cutting on the yield and chemical composit Nigerian fodder grasses (Tripsacum laxicum Nash) W.A.J. Biol. Chem.4 (3): 46

Passoni, F.; Rosemberg, M. y Flores Mere, A. 1992. Evaluación de gramíneas y leguminosas forrajeras en Satipo, Perú. Pasturas Tropicales 14(1):32-34.

Ramírez, O.; Hernández, A.; Carneiro da Silva, S.; Pérez, J.; Enríquez, J.; Quero, A.; Herrera, J. y Cervantes, A. 2009. Acumulación de forraje, crecimiento y características estructurales del pasto Mombaza (Panicum maximum Jacq.) cosechado a diferentes intervalos de corte. Técnica Pecuaria en México 47(2):203-213.

Reynoso, O.; Garay, A.; Da Silvac, S.; Pérez, J.; Quiroz, J.; Carrilloe, A.; Haro, G. y Núñe, A. 009. Acumulación de forraje, crecimiento y características estructurales del pasto Mombaza (Panicum maximum Jacq.) cosechado a diferentes intervalos de corte. Téc Pecu Méx 47(2):203-213. Recuperado de: file://C:/Users/ of $\% 20$ Investig/Downloads/art7.pdf

Ruiz, F.; Rodríguez, E.; Pinzón, J.; Anzola, H. y Castro, L. 2015. Establecimiento y evaluación del género Panicum maximum cv Massai en la hacienda Guachicono del Bordo, Patía (Cauca). Revista Ciencia Animal 9:125154.

Sánchez, P.A. y Salinas, J.G.1993. Suelosácidos. Estrategias para su manejo con bajos insumos en Ámerica Tropical. Bogotá, Colombia. Editor Sociedad Colombiana de la Ciencia de Suelo. 93 p.

Segura, M. y Chamblee, D. 1967. Forrajes en el Perú. Centro Regional de Ayuda Técnica, Agencia para el Desarrollo Internacional (AID), México.

Skerman, P.J. y Riveros, F. 1992. Gramíneas Tropicales. FAO, Roma, Italia.

Zanini, G.; Santos, G. and Sbrissia, A. 2012. Frequencies and intensities of defoliation in Aruana Guineagrass swards: accumulation and morphological composition of forage. Revista Brasileira de Zootecnia 41(4): $905-$ 913. 